movement, "Saint Peter's Square, noon; the immensity of space," featured toccata-like figures in the cello, into which a growing electronic simultaneity was introduced as a background, followed by evocative restatements of an earlier piano motif.

The final work on the concert was a third video piece: Cylindrical

Dimensions by Michael Rhoades. Audio was created using Csound, and video using Maya, the whole being presented as "a visual music composition," as "an exploration of the effects of reflective curvatures in light." Three-dimensional representations of rotating aquamarine string-like figures against a monochrome background gave way to a monochrome, rotating survey of the overall visual texture. Lavender, then blue colors were introduced, transitioning to a shimmering blue, then green. As a formal component, the piece became more sonically active and visually flashing as it moved through a series of sound events produced by means of generative algorithms created by the composer. The piece culminated with a rapid, palindromic return through its scenic types.

\section{Installation Reviews}

Haein Kang: Illusion: you can hear, but you cannot see (2018) NYU Blackbox Theater, June 19, 2019 Reviewed by Teresa Marie Connors

Haein Kang's performance-based installation, Illusion: you can hear, but you cannot see, combinations hightech equipment + code with video projection and eight customized percussion instruments. Each instrument is equipped with an up and down mechanical moving arm device, elastic string attached to the arm, and a fishing line weight at the end of the string. Each weight hovers above a material object made from either metal, glass or wood. When the mechanical arm is triggered to move, each weight strikes their respective object causing it to sound.

What triggers the movement of the mechanical elements are alpha brain waves. For the ICMC2019 performance-based installation, Kang sat in the middle of the semi-circularly placed instruments wearing an EEG headset. A customized computer program transcoded the data from her 
headset, which then trigger each percussion module. The activation was specific to Kang's eyes being closed, which activates the 8 to $13 \mathrm{~Hz}$ electrical signal that occurs in the visual cortex. Directly behind Kang was a video projection which was also triggered by the EEG headset, and comprised of either a close-up photo of an eye opened or closed.

During Kang's performance at the NYU Blackbox Theater, it took time for the equipment to work correctly. But, as any creative practitioner working with code + equipment can attest, it's challenging to get everything to work. Audience members could see Kang sitting in the center of the room wearing the EEG headset and surrounded by the customized percussion instruments, but nothing happened. Once Kang rebooted the system, and all was running properly, the combination of brain-computer interface, customized percussion instruments and video project were fascinating and an elegant use of the various materials and processes.

Examples of Illusion: you can hear, but you cannot see are available on Kang's web page: www.haeinkang.org/gallery-4culture

Daniel B. Formo: The Orchestra of Speech (2018)

NYU Library, June 17-22, 2019

Reviewed by Teresa Marie Connors

\section{Daniel Formo's The Orchestra of}

Speech is a well-constructed sound + instrument installation. Developed during his $\mathrm{PhD}$ research at the Norwegian University of Science and Technology in Trondheim, Formo's project explores the relationship between everyday speech and musical improvisation and is concerned with human speech gestures as performative material.

Visitors to the ICMC 2019 rendition of the work found percussion and string instruments situated and hung throughout a medium-sized room. Mounted on each instrument is a transducer that receives speech analysis from a custom-made software created in Max. The software extracts in real-time musical content of prerecorded and live speech. The live input occurs via an old rotary telephone located in the center of the room. Visitors to the installation are encouraged to interact with the 
phone when it rings, which are, in turn, recorded, analyzed and routed to the acoustic instruments via the respective transducers.

For the speech analysis, Formo focuses on the prosodic qualities of speech such as strong accents, intonation and rhythm. Routing the resulting abstractions to the transducerinstruments produces a delicate musical world with the use of drum skin, cymbal metal, guitar and zither. Moving around the room to individual instruments, it becomes apparent the musical material is produced from speech gestures and, with the addition of the live telephone input, interaction with The Orchestra of Speech can become an improvisatory process. Formo's complete project is located at

http://orchestraofspeech.com

\section{Alexis S Crawshaw: Kroumatograph}

No. 4 (2019)

Fridman Gallery, June 17-23, 2019

Reviewed by Teresa Marie Connors

\section{Alexis S Crawshaw's Kroumatograph}

No. 4 explores the use of lowfrequency static additive tone $(\sim 30-80 \mathrm{~Hz})$ to construct this site- specific composition-installation. Visitors to the gallery space are invited to follow a path score mapped out on the floor, which also includes designated stopping points. While walking the score, subtle frequencybeating polyrhythmic shifts occur as you move to different locations in the room. Crawshaw notes that using theses frequencies between $\sim 30-80 \mathrm{~Hz}$ adds a haptic element to the work, as they correspond to the general frequency range of the chest resonance in the human body.

Running between ICMC shows, it took time to slow down to experience the sonic world Kroumatograph No. 4 inhabits. After two score walks, a meditative state emerged which motivated a third and subsequent free improvisatory stroll around the room. Being embedded in this sonic world is captivating as you can feel the vibrations in the body.

Crawshaw's broader research is concerned with the impact of infrasounds in architectural structures and the potential effects on those that reside within. Kroumatograph No. 4 brings to the foreground this sonic landscape in a well thought out 
composition-installation.

Mara Helmuth, Yunze Mu, Owen Hopper, Zhixin Xu, Shawn Milloway: Five Worlds NYU Library, June 17, 2019, 3 p.m. Reviewed by Wing-Lam Sin

Five Worlds is an immersive virtual reality game-like adventure. After wearing the HTC Vive VR headset, audience will start their journey of Five Worlds. Starting point is a peaceful universe, which can be seen via the headset.

The audience can use the handheld controller to move and touch different spheres, each opening up a completely different environment another world to explore.

The worlds in Five Worlds are completely different concerning visual and audio components, but also regarding the interactive features that enable the audience to experience the diverse environments within a single installation.

The five worlds include "Tranquilarea", a peaceful universe with a golden Menger Sponge by Mara Helmuth; "Wood Sounds World", a relaxing natural habitat by Owen Hopper;
"Idiophone", a world with an interactive musical instrument by Zhixin Xu; "Visualizer Cave”, a cave with different sonic visualizers by Shawn Milloway; and "Sky World", a world with a beautiful sunrise by Yunze Mu.

There are a lot of interactive components in each world which encourage the player to explore and discover different hidden sounds that are combined with interesting visuals. For example, when getting close to ponds in "Wood Sounds World", a calm music of running water and ripples will start playing. When getting close to a firefly-like sonic visualizer in "Visualizer Cave", a bunch of "fireflies" fly away with a dreamy music. Players can also control through verbal commands, e.g. to fly or trigger an action. The sound effects and background music are synchronizing in time with the visual effects and the player's actions, which create a unique and impressive experience. 